\title{
The threshold contrast thickness evaluated with different CDMAM phantoms and software
}

\author{
Ewa Fabiszewska, \\ Iwona Grabska, \\ Katarzyna Pasicz
}

\begin{abstract}
The image quality in digital mammography is described by specifying the thickness and diameter of disks with threshold visibility. The European Commission recommends the CDMAM phantom as a tool to evaluate threshold contrast visibility in digital mammography [1, 2]. Inaccuracy of the manufacturing process of CDMAM 3.4 phantoms (Artinis Medical System BV), as well as differences between software used to analyze the images, may lead to discrepancies in the evaluation of threshold contrast visibility. The authors of this work used three CDMAM 3.4 phantoms with serial numbers 1669, 1840, and 1841 and two mammography systems of the same manufacturer with an identical types of detectors. The images were analyzed with EUREF software (version 1.5.5 with CDCOM 1.6. exe file) and Artinis software (version 1.2 with CDCOM 1.6. exe file). The differences between the observed thicknesses of the threshold contrast structures, which were caused by differences between the CDMAM 3.4 phantoms, were not reproduced in the same way on two mammography units of the same type. The thickness reported by the Artinis software (version 1.2 with CDCOM 1.6. exe file) was generally greater than the one determined by the EUREF software (version 1.5.5 with CDCOM 1.6. exe file), but the ratio of the results depended on the phantom and diameter of the structure. It was not possible to establish correction factors, which would allow correction of the differences between the results obtained for different CDMAM 3.4 phantoms, or to correct the differences between software. Great care must be taken when results of the tests performed with different CDMAM 3.4 phantoms and with different software application are interpreted.
\end{abstract}

Key words: CDMAM phantom • digital mammography • threshold visibility

E. Fabiszewska, I. Grabska ${ }^{\bowtie}$, K. Pasicz

Medical Physics Department,

The Maria Skłodowska-Curie Memorial Cancer Center and Institute of Oncology,

5 W. K. Roentgena Str., 02-781 Warsaw, Poland,

Tel.: +48 22546 2954, Fax: +48 22644 9182,

E-mail: i.grabska@zfm.coi.pl

Received: 18 June 2014

Accepted: 17 August 2015

\section{Introduction}

The European Commission recommends the CDMAM phantom as a tool to evaluate threshold contrast visibility in digital mammography $[1,2]$. The use of the CDMAM phantom for that purpose has been proposed by Young [3] and other researchers. Recently, the phantom has been criticized because of poor reproducibility of the manufacturing process $[4,5]$, but it is still recommended by the European Commission. According to the manufacturer's information [6], "(...) during vaporization of the gold disks, the aluminium plate is covered with a mask with lasered holes of the desired diameter. Due to small irregularities of the mask and specific deviations related to the vaporization process, the disks can deviate slightly from the intended diameter, and will not be exactly circular. Also the thickness of the disks may deviate from the indicated values, mainly due to the gold vaporization profile. Thickness at the centre of the phantom tends to be more, and at the edges less than intended (...). Generally, deviations appear to become more significant at smaller disk diameter (up to $5 \%$ in area and $10 \%$ in diameter). Thickness deviations from indicated values appear to be $<10 \%$." 
The image quality in digital mammography is described by specifying the thickness and diameter of disks with threshold visibility. In the original method, visibility was determined visually by a group of three human observers [1]. However, studies have shown the inter- and intraobserver variability in the assessment of the structures in the CDMAM phantom images $[7,8]$. To eliminate the influence of the human factor, several computer programs to analyze the CDMAM phantom images have been developed. Computer analysis of the images is currently recommended by the European Commission [2]. Automatic readout of the CDMAM phantom images provides a reliable and less time-consuming alternative to human readout [9].

The CDMAM analyser software (later referred to as 'Artinis software'), offered commercially by the manufacturer of the CDMAM phantom (Artinis Medical Systems BV), is an example of this kind of software. Another one is the Guildford CDMAM Analyser (later referred to as 'EUREF software'), which is freely available on the website of the European Reference Organisation for Quality Assured Breast Screening and Diagnostic Services. The EUREF software uses the CDCOM exe file [10] as a core component and performs the analysis with methods described by Young et al. [3]. The Artinis software uses the CDCOM exe file, as well as the EUREF software. However, there are some differences between these two software applications $[6,11]$.

The inaccuracy of the manufacturing process of CDMAM 3.4 phantoms, as well as the differences between software used to analyze the images, may lead to discrepancies in the evaluation of threshold contrast visibility. Therefore, the authors of this work used several CDMAM 3.4 phantoms and two identical mammography systems to answer the following questions.

- Are the differences between CDMAM 3.4 phantoms reproduced in the same way on different mammography systems?

- What are the differences between results of threshold contrast visibility analysis performed with Artinis software and EUREF software?

- Is it possible to establish correction factors that would allow to correct the results for differences between different phantoms and software?

\section{Materials and methods}

\section{Equipment}

Three CDMAM 3.4 phantoms (Artinis Medical System BV) with serial numbers 1669,1840 , and 1841 were used. Images of the phantoms were acquired on two Siemens Mammomat Inspiration mammography units, with serial numbers 3420 (later referred to as mammography unit 1) and 3419 (mammography unit 2). Both units were manufactured in 2011 and installed at the same hospital. Both mammography units were equipped with an identical types of a flat panel direct conversion digital detectors, consisting of amorphous selenium (a-Se) in conjunction with a thin film transistor readout array. The European requirements [1] for technical and physical parameters were met for both units.

\section{Images of the CDMAM 3.4 phantoms}

The phantoms were positioned on the bucky of the mammography unit. The structures with the smallest diameter were located closest to the chest side of the bucky [6]. Poly (methyl metacrylate) (PMMA) blocks $20 \mathrm{~mm}$ thick were placed below and above the CDMAM 3.4 phantom. Total attenuation of the X-ray beam caused by the phantom with PMMA blocks was then equivalent to that caused by a layer of typical breast tissue $60 \mathrm{~mm}$ thick. Each of the CDMAM 3.4 phantoms was equipped by the manufacturer with its own set of the PMMA blocks. To eliminate the influence of the potential differences between different sets of blocks, only one of them (serial no. 1669) was used with all of the phantoms.

A set of 32 images was acquired for each of the CDMAM 3.4 phantoms and each of the mammography units. A relatively large number of images improves the reproducibility of the analysis process for all detail sizes [7]. All of the images were obtained in an unprocessed study (QC-RAW) for identical exposure parameters: $28 \mathrm{kV}, 140 \mathrm{mAs}$ (manual mode), W/Rh anode/filter combination, with an antiscatter grid. The exposure parameters were equivalent to those chosen for a $50 \mathrm{~mm}$ layer of PMMA by the automatic exposure control system working in OPDOSE mode, which is routinely used for examination of women. For both mammography units, the half value layer for the W/Rh anode/filter combination for $28 \mathrm{kV}$ was $0.57 \mathrm{~mm}$. The mean glandular dose for a $5.0 \mathrm{~cm}$ PMMA was of $1.2 \mathrm{mGy}$ for both mammography units.

Once all the required images were taken, they were transferred to a disc for subsequent analysis at our laboratory.

\section{Analysis of the images}

The images were analyzed with the EUREF software (the 1.5.5 version with CDCOM 1.6. exe file). Results of the analysis were obtained separately for each diameter of the structures $(0.10,0.13,0.16$, $0.20,0.25,0.31,0.40,0.50,0.63,0.80$ and $1.00 \mathrm{~mm})$. The results were expressed as automatic threshold gold thickness (ATGT), fit to predicted gold thickness (FTPGT), and 2SE (all values expressed in $\mu \mathrm{m}$ ), as shown in Table 1 . The analysis of the images with the EUREF software involved the following steps:

- the detection of structures with the CDCOM 1.6. exe file;

- combining the results obtained for the series of images (32 images in our study) and the smoothing of the detection matrix with a Gauss filter, as shown in Fig. 1;

- fitting of the psychometric curve (describing the dependence of the proportion of correctly detected structures to the nominal thickness of structures) [12] and the estimation of the thickness of 
Table 1. Exemplary final results given by the EUREF software

\begin{tabular}{|c|c|c|c|c|c|c|c|c|c|c|c|c|c|c|c|c|}
\hline \multicolumn{2}{|c|}{$\begin{array}{l}\text { Diameter } \\
{[\mathrm{mm}]}\end{array}$} & \multicolumn{4}{|c|}{$\begin{array}{l}\text { Automatic threshold } \\
\text { gold thickness }\end{array}$} & \multicolumn{4}{|c|}{$\begin{array}{l}\text { Predicted human } \\
\text { gold thickness }\end{array}$} & \multicolumn{5}{|c|}{$\begin{array}{l}\text { Fit to predicted } \\
\text { gold thickness }\end{array}$} & \multicolumn{2}{|c|}{$2 \mathrm{SE}$} \\
\hline 0.08 & & \multicolumn{4}{|c|}{1.30} & \multicolumn{4}{|c|}{1.95} & \multicolumn{4}{|c|}{1.67} & \multicolumn{3}{|c|}{0.21} \\
\hline 0.10 & & \multicolumn{4}{|c|}{0.66} & \multicolumn{4}{|c|}{1.03} & \multicolumn{4}{|c|}{1.03} & \multicolumn{3}{|c|}{0.12} \\
\hline 0.13 & & \multicolumn{4}{|c|}{0.40} & \multicolumn{4}{|c|}{0.66} & \multicolumn{4}{|c|}{0.61} & \multicolumn{3}{|c|}{0.07} \\
\hline 0.16 & & \multicolumn{4}{|c|}{0.26} & \multicolumn{4}{|c|}{0.43} & \multicolumn{4}{|c|}{0.43} & \multicolumn{3}{|c|}{0.05} \\
\hline 0.20 & & \multicolumn{4}{|c|}{0.17} & \multicolumn{4}{|c|}{0.29} & \multicolumn{4}{|c|}{0.31} & & & .04 \\
\hline 0.25 & & & & 0.11 & & & & .20 & & & & 0.23 & & & & .03 \\
\hline 0.31 & & & & 0.09 & & & & .17 & & & & 0.18 & & & & .02 \\
\hline 0.40 & & & & 0.08 & & & & .16 & & & & 0.15 & & & & .02 \\
\hline 0.50 & & & & 0.07 & & & & .14 & & & & 0.12 & & & & .02 \\
\hline 0.63 & & & & 0.05 & & & & .10 & & & & 0.10 & & & & .01 \\
\hline 0.80 & & & & 0.04 & & & & .09 & & & & 0.09 & & & & .01 \\
\hline 1.00 & & & & 0.03 & & & & .07 & & & & 0.08 & & & & .01 \\
\hline & & & & & & & Sm & ooth D & ata & & & & & & & \\
\hline & propor & tion of & correc & tly dete & ected o & iscs & & & & & & & & & & \\
\hline & gold th & icknes & $s($ um) & & & & & & & & & & & & & \\
\hline diam & 0.03 & \begin{tabular}{l|l|}
0.04 \\
\end{tabular} & 0.05 & \begin{tabular}{|c|}
0.06 \\
\end{tabular} & \begin{tabular}{|c|}
0.08 \\
\end{tabular} & $\begin{array}{l}0.1 \\
\end{array}$ & 0.13 & 0.16 & 0.2 & 0.25 & 0.36 & 0.5 & 0.71 & 1.00 & 1.42 & 2.00 \\
\hline 2.00 & & 0.925 & 0.917 & 0.938 & 0.979 & 1.000 & 1.000 & 1.000 & 1.000 & 1.000 & & & & & & \\
\hline 1.6 & 0.931 & 0.888 & 0.895 & 0.953 & 0.992 & 1.000 & 1.000 & 1.000 & 1.000 & 1.000 & 1.000 & & & & & \\
\hline 1.25 & 0.849 & 0.836 & 0.871 & 0.930 & 0.969 & 0.992 & 1.000 & 1.000 & 1.000 & 1.000 & 1.000 & 1.000 & & & & \\
\hline 1.00 & 0.677 & 0.719 & 0.785 & 0.859 & 0.934 & 0.984 & 1.000 & 1.000 & 1.000 & 1.000 & 1.000 & 1.000 & 1.000 & & & \\
\hline 0.8 & 0.547 & 0.594 & 0.691 & \begin{tabular}{|l|}
0.797 \\
\end{tabular} & 0.883 & 0.953 & 0.992 & 1.000 & 1.000 & 1.000 & 1.000 & 1.000 & 1.000 & 1.000 & & \\
\hline \begin{tabular}{l|}
0.63 \\
\end{tabular} & 0.516 & 0.547 & 0.629 & 0.695 & 0.758 & 0.879 & 0.977 & 1.000 & 1.000 & 1.000 & 1.000 & 1.000 & 1.000 & 1.000 & 1.000 & \\
\hline 0.5 & 0.443 & 0.453 & 0.488 & 0.535 & 0.621 & 0.785 & 0.922 & 0.977 & 0.996 & 1.000 & 1.000 & 1.000 & 1.000 & 1.000 & 1.000 & 1.000 \\
\hline 0.4 & 0.339 & 0.316 & 0.363 & \begin{tabular}{|l|}
0.453 \\
\end{tabular} & 0.574 & 0.730 & 0.840 & 0.918 & 0.973 & 0.988 & 0.996 & 1.000 & 1.000 & 1.000 & 1.000 & 1.000 \\
\hline $\begin{array}{ll}0.31 \\
\end{array}$ & 0.300 & 0.292 & 0.379 & \begin{tabular}{|l|}
0.465 \\
\end{tabular} & 0.566 & 0.711 & 0.801 & 0.855 & 0.898 & 0.945 & 0.988 & 1.000 & 1.000 & 1.000 & 1.000 & 1.000 \\
\hline 0.25 & & 0.313 & 0.358 & 0.402 & 0.480 & 0.609 & 0.742 & 0.789 & 0.781 & 0.867 & 0.973 & 1.000 & 1.000 & 1.000 & 1.000 & 1.000 \\
\hline 0.2 & & & 0.250 & 0.313 & 0.391 & 0.473 & 0.598 & 0.645 & 0.629 & 0.750 & 0.906 & 0.961 & 0.98 & 0.996 & 1.000 & 1.000 \\
\hline 0.16 & & & & 0.250 & 0.321 & 0.371 & 0.418 & 0.449 & 0.488 & 0.617 & 0.770 & 0.859 & 0.926 & 0.980 & 1.000 & 1.000 \\
\hline 0.13 & & & & & 0.267 & 0.288 & 0.262 & 0.309 & 0.418 & 0.496 & 0.578 & 0.695 & 0.809 & 0.914 & 0.984 & 1.000 \\
\hline 0.1 & & & & & & 0.25 & 0.208 & 0.246 & 0.348 & 0.383 & 0.410 & 0.531 & 0.652 & 0.766 & 0.875 & 0.927 \\
\hline 0.08 & & & & & & & 0.256 & 0.263 & 0.301 & 0.324 & 0.340 & 0.406 & 0.473 & 0.547 & 0.638 & 0.744 \\
\hline \begin{tabular}{l|l|}
0.06 \\
\end{tabular} & & & & & & & & 0.294 & 0.318 & \begin{tabular}{|l|}
0.328 \\
\end{tabular} & 0.313 & 0.292 & 0.302 & 0.370 & 0.413 & \\
\hline
\end{tabular}

Fig. 1. Exemplary data of smoothing of the detection matrix with a Gauss filter.

structure for which proportion of structures was detected correctly, which is equal to $62.5 \%$, as shown in Fig. 2a and Fig. 2b, respectively;

- scaling up the results obtained with the software to values for a typical human observer for each detail diameter, which are predicted using following equation:

$$
\mathrm{TC}_{\text {predicted }}=r \mathrm{TC}_{\text {auto }}
$$

where $\mathrm{TC}_{\text {predicted }}$ is the predicted threshold contrast for a typical observer and $\mathrm{TC}_{\text {auto }}$ is the threshold contrast measured using an automated procedure with CDMAM images. The contrasts were calculated from gold thickness for a nominal tube

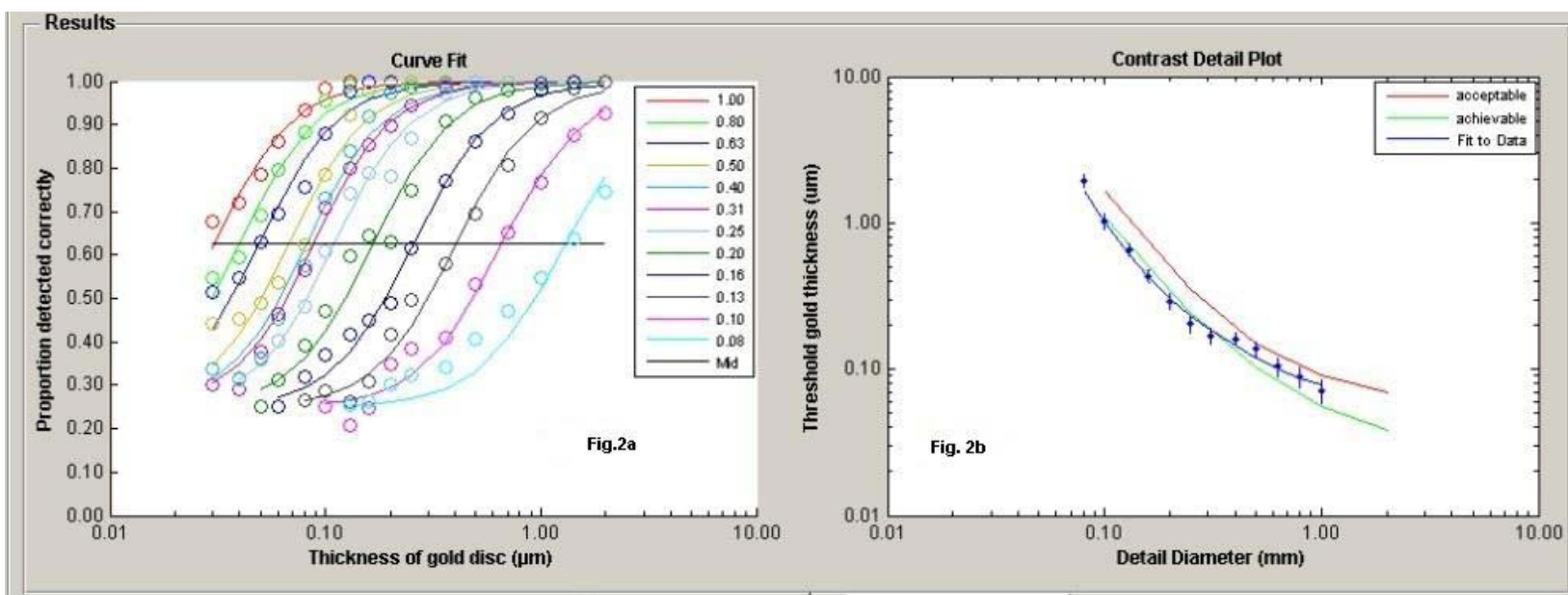

Fig. 2. Exemplary results of the analysis of the images with EUREF software: (a) fitting of the psychometric curve, (b) estimation of the threshold gold thickness of structure for which proportion of correctly detected structures is equal to $62.5 \%$. 
Table 2. Values of $r$ used to predict threshold contrast [11] Diameter of gold disc
$[\mathrm{mm}]$
Average ratio of human to automatically measured threshold contrast: $r$

\begin{tabular}{ll}
\hline 0.08 & 1.40 \\
0.10 & 1.50 \\
0.13 & 1.60 \\
0.16 & 1.68 \\
0.20 & 1.75 \\
0.25 & 1.82 \\
0.31 & 1.88 \\
0.40 & 1.94 \\
0.50 & 1.98 \\
0.63 & 2.01 \\
0.80 & 2.06 \\
1.00 & 2.11 \\
\hline
\end{tabular}

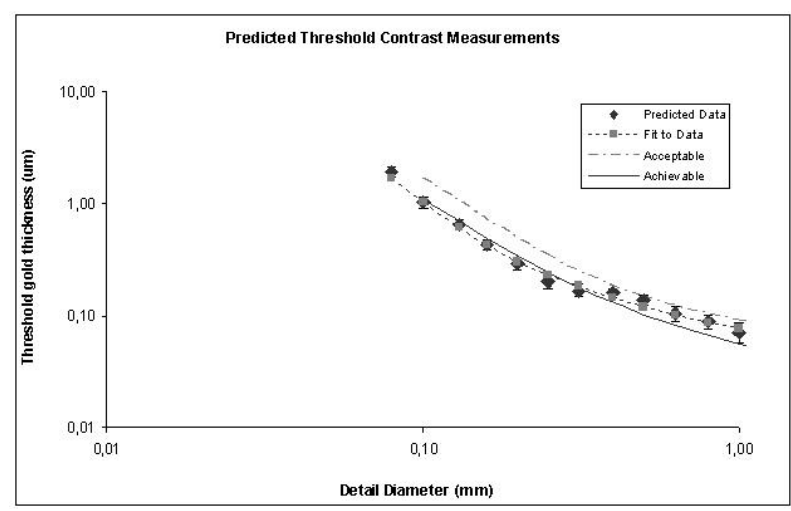

Fig. 3. Fitting of the third degree polynomial to the dependence of predicted threshold contrast for a typical observer on diameter of structures. voltage of $28 \mathrm{kV}$ and a Mo/Mo target filter combination as described in the European protocol; $r$ is the average ratio between human and automatic threshold contrast determined experimentally with the values shown in Table 2;

- fitting of the third degree polynomial to the dependence of threshold gold thickness on the diameter of structures, as shown in Fig. 3.

The ATGT values were obtained after the first step of the analysis, which was after the detection of the structures and the smoothing of the detection matrix only with the Gauss filter. The FTPGT values were the final result of the analysis with the EUREF software, and included several steps of analysis, such as the fitting of the psychometric curve (describing the dependence of the percentage of detected structures on their nominal thickness) [11] and the fitting of the third degree polynomial. The FTPGT values were reported by the software together with the information on the total expanded uncertainty at 95\% confidence level (2SE). The 2SE uncertainty was calculated as a quotient between the standard deviation of the FTPGT values obtained for the 32 analyzed images, and the square root of the number of the images.

Images of the three phantoms obtained on mammography unit 1 were additionally analyzed with the Artinis software (version 1.2 with CDCOM 1.6. exe file). The results were expressed as the thickness of a structure with threshold contrast, for the same set of diameters as with the EUREF software. The analysis performed with the Artinis software did not include the smoothing of the detection matrix with the Gauss filter, the psychometric curve fitting, and fitting of the third degree polynomial to dependence of threshold gold thickness on diameter of structures

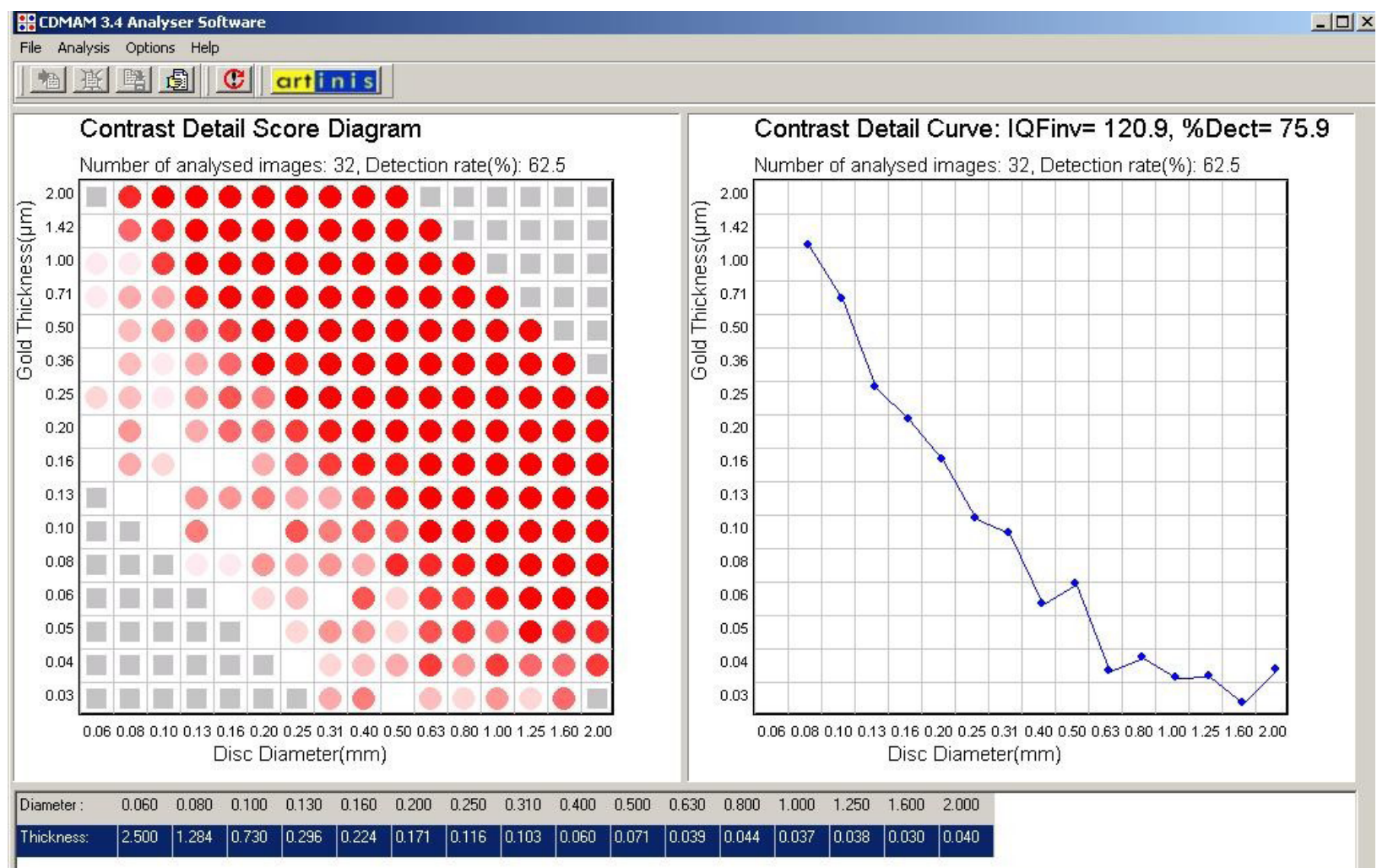

Fig. 4. Exemplary results of the analysis with Artinis software, number of analyzed images of CDMAM 3.4 phantom: 32. 
Table 3. The FTPGT values for three CDMAM 3.4 phantoms (serial nos. 1669, 1840, and 1841) and two mammography units. Total expanded uncertainty at $95 \%$ confidence level is also presented (2SE)

\begin{tabular}{lcccccc}
\hline & \multicolumn{7}{c}{ FTPGT \pm 2SE $[\mu \mathrm{m}]$} \\
\cline { 2 - 7 } $\begin{array}{c}\text { Nominal } \\
\text { diameter } \\
\text { of structure } \\
{[\mathrm{mm}]}\end{array}$ & \multicolumn{7}{c}{ Mammography unit 1} & \multicolumn{3}{c}{ Mammography unit 2} \\
\cline { 2 - 7 } & 1669 & 1840 & 1841 & 1669 & 1840 & 1841 \\
\hline 0.08 & $1.666 \pm 0.079$ & $1.565 \pm 0.074$ & $1.455 \pm 0.069$ & $1.812 \pm 0.086$ & $1.690 \pm 0.080$ & $1.411 \pm 0.067$ \\
0.10 & $1.041 \pm 0.047$ & $0.983 \pm 0.044$ & $1.005 \pm 0.045$ & $1.099 \pm 0.050$ & $1.053 \pm 0.048$ & $0.953 \pm 0.043$ \\
0.13 & $0.627 \pm 0.025$ & $0.600 \pm 0.024$ & $0.654 \pm 0.027$ & $0.642 \pm 0.026$ & $0.636 \pm 0.026$ & $0.614 \pm 0.025$ \\
0.16 & $0.441 \pm 0.018$ & $0.428 \pm 0.017$ & $0.475 \pm 0.019$ & $0.444 \pm 0.018$ & $0.451 \pm 0.018$ & $0.447 \pm 0.018$ \\
0.20 & $0.314 \pm 0.014$ & $0.310 \pm 0.014$ & $0.341 \pm 0.015$ & $0.312 \pm 0.014$ & $0.324 \pm 0.015$ & $0.324 \pm 0.015$ \\
0.25 & $0.233 \pm 0.010$ & $0.234 \pm 0.010$ & $0.251 \pm 0.011$ & $0.231 \pm 0.010$ & $0.243 \pm 0.011$ & $0.242 \pm 0.011$ \\
0.31 & $0.180 \pm 0.008$ & $0.184 \pm 0.008$ & $0.191 \pm 0.008$ & $0.179 \pm 0.008$ & $0.190 \pm 0.008$ & $0.187 \pm 0.008$ \\
0.40 & $0.137 \pm 0.006$ & $0.141 \pm 0.006$ & $0.143 \pm 0.006$ & $0.137 \pm 0.006$ & $0.145 \pm 0.006$ & $0.142 \pm 0.006$ \\
0.50 & $0.109 \pm 0.005$ & $0.114 \pm 0.006$ & $0.114 \pm 0.006$ & $0.111 \pm 0.005$ & $0.117 \pm 0.006$ & $0.114 \pm 0.006$ \\
0.63 & $0.087 \pm 0.005$ & $0.092 \pm 0.005$ & $0.093 \pm 0.005$ & $0.091 \pm 0.005$ & $0.094 \pm 0.005$ & $0.092 \pm 0.005$ \\
0.80 & $0.071 \pm 0.004$ & $0.075 \pm 0.004$ & $0.078 \pm 0.005$ & $0.076 \pm 0.004$ & $0.077 \pm 0.004$ & $0.077 \pm 0.004$ \\
1.00 & $0.059 \pm 0.004$ & $0.063 \pm 0.004$ & $0.069 \pm 0.005$ & $0.065 \pm 0.004$ & $0.064 \pm 0.004$ & $0.067 \pm 0.004$ \\
\hline
\end{tabular}

[6]. Exemplary results of the analysis of 32 images with Artinis software are presented in Fig. 4.

\section{Comparison of the results}

For each of the mammography units, the differences between the FTPGT values obtained with different pairs of phantoms (1840 and 1669, 1841 and 1669, 1841 and 1840) were compared. The 2 SE values were included in the comparison. Moreover, the differences between the FTPGT values obtained for the first and the second phantoms were expressed as a percentage of the result obtained for the second phantom.

The differences between the thickness of the structures obtained by the Artinis software (version 1.2 with CDCOM 1.6. exe file) and the ATGT values obtained by the EUREF software (version 1.5 .5 with CDCOM 1.6. exe file) were also calculated for each of the phantoms. The differences were expressed as a percentage of the ATGT values.

\section{Results and discussion}

The FTPGT values for three CDMAM 3.4 phantoms and two mammography units

The FTPGT values for three CDMAM 3.4 phantoms and two mammography units are given in Table 3, together with the uncertainty (2SE).

The differences obtained for different pairs of phantoms for both mammography units were not equal within the uncertainty limits [13] for structures with a nominal diameter of:

- $0.08,0.10$, and $0.40 \mathrm{~mm}$ for the pair of phantoms 1840 and 1669 ,

- $0.08,0.10$, and $0.13 \mathrm{~mm}$ for the pair of phantoms 1841 and 1669 ,

- $0.08,0.10,0.13,0.16$, and $0.20 \mathrm{~mm}$ for the pair of phantoms 1841 and 1840 .

Moreover, these differences were not identical for both units (Fig. 5).
The differences between the FTPGT values obtained for the different pairs of phantoms are presented in Fig. 5. For each pair of phantoms (1840 and 1669,1841 and 1669, 1841 and 1840), the differences between FTPGT values obtained for the first and the second phantoms were expressed as a percentage of the result obtained for the second phantom. The observed differences for each pair of phantoms were not the same for both mammography units. In most cases, the calculated differences were larger for the mammography unit 1 (Fig. 5b and 5c). The differences were larger for the mammography unit 2 for the pair of phantoms 1840 and 1669 and the structures with diameters from 0.10 to $0.50 \mathrm{~mm}$ (Fig. 5a).

The results presented show that it is not possible to establish a relationship between FTPGT obtained for different CDMAM 3.4 phantoms, as the relationship differs between different mammography systems. Any comparison of image quality described by the diameter and thickness of the threshold contrast structure in the CDMAM 3.4 phantom between different mammography systems seems difficult and may lead to erroneous results of the test.

The observed differences between the results obtained with different phantoms reach $12 \%$ (pair of phantoms 1841 and 1840, Fig. 5c) and may lead to overestimation of the threshold thickness of the structure with $0.10 \mathrm{~mm}$ diameter. This thickness is used to establish the criteria for the "object thickness and tube voltage compensation" test, which is used to evaluate performance of the automatic exposure control system $[1,2]$. The differences between phantoms may then lead to erroneous results for that test as well.

\section{Differences between the results obtained with the Artinis software and the EUREF software}

In the vast majority of cases, the thickness of the threshold contract structure given by the Artinis software was bigger than the ATGT given by the EUREF software, with the maximum observed difference of 39\% of the ATGT (Table 4). Only for one 
a

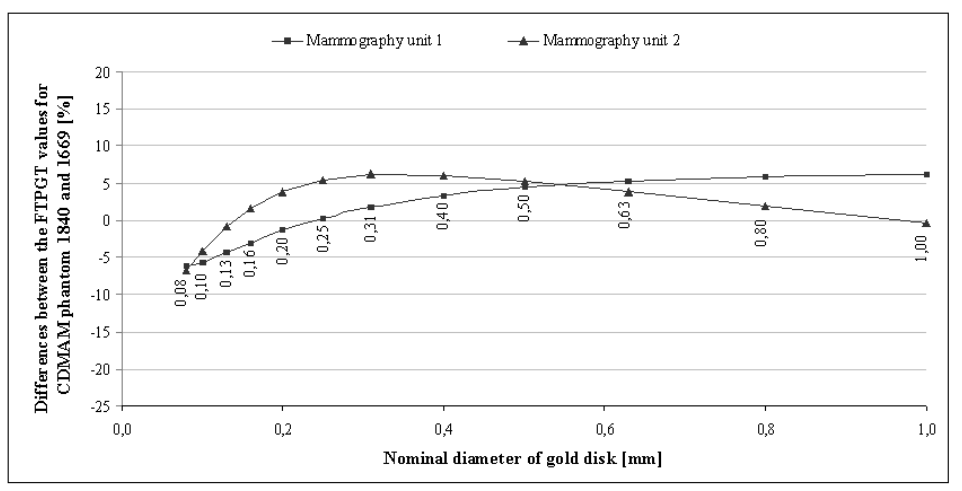

$\mathrm{b}$
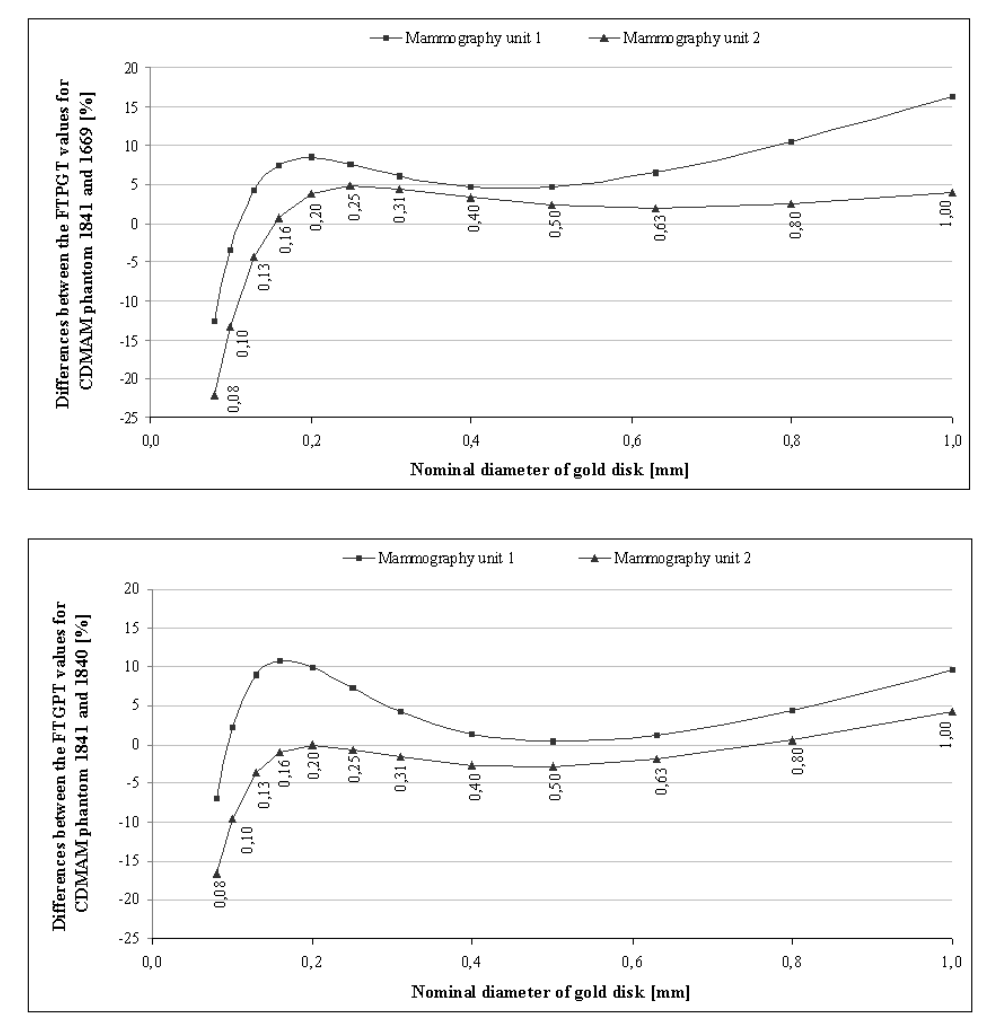

Fig. 5. Differences between the FTPGT values obtained with different CDMAM 3.4 phantoms: (a) 1840 and 1669 , (b) 1841 and 1669, (c) 1841 and 1840, expressed as a percentage of the result obtained with the second phantom. Discrete data points are connected with lines for illustrative purposes.

Table 4. Differences between the thickness of the threshold contrast structure given by the Artinis software and the ATGT values given by the EUREF software for three CDMAM 3.4 phantoms, expressed as a percentage of the result obtained with the EUREF software

\begin{tabular}{lrrr}
\hline \multirow{2}{*}{$\begin{array}{c}\text { Nominal diameter } \\
\text { of structure } \\
{[\mathrm{mm}]}\end{array}$} & \multicolumn{2}{c}{$\begin{array}{c}\text { Differences between thickness of the threshold contrast structure given by } \\
\text { the Artinis software and the ATGT values given by the EUREF software [\%] }\end{array}$} \\
\cline { 2 - 4 } & 1669 & 1840 & 1841 \\
\hline 0.10 & 20 & 13 & 8 \\
0.13 & 9 & 5 & 23 \\
0.16 & 15 & 9 & -4 \\
0.20 & 11 & 5 & 7 \\
0.25 & 7 & 18 & 16 \\
0.31 & 20 & 2 & 8 \\
0.40 & 12 & 19 & 1 \\
0.50 & 18 & 24 & 24 \\
0.63 & 17 & 16 & 19 \\
0.80 & 39 & 17 & 30 \\
1.00 & 26 & & 1 \\
\hline
\end{tabular}


data point (phantom 1841, structure with $0.16 \mathrm{~mm}$ diameter) was the thickness of structure given by the Artinis software slightly smaller than the ATGT (by $4 \%$ of the ATGT).

The EUREF software in the first stage of the analysis smoothed the data by applying a Gaussian function [3] while the Artinis software does not apply any smoothing of the data. Moreover, the Artinis software does not fit psychometric curve and third degree polynomial to dependence of threshold gold thickness on the diameter of structures. This is the reason for the differences between the results received by the two software applications analyzed. The differences between the results obtained using the two programs were not the same for each phantom CDMAM (Table 4). This was due to differences (shown in the previous section) between the CDMAM 3.4 phantoms.

Unfortunately, the relationship in the results obtained between the two software packages is not obvious and was different for different phantoms. Therefore, it was not possible to establish correction factors, which would enable the correction of the differences between the software applications.

\section{Conclusions}

The differences between the observed thicknesses of the threshold contrast structures, which are caused by differences between the CDMAM 3.4 phantoms (Artinis Medical System BV), were not reproduced in the same way on two mammography units of the same type. The thickness reported by the Artinis software (version 1.2 with CDCOM 1.6. exe file) was generally greater than that obtained by the EUREF software (version 1.5.5 with CDCOM 1.6. exe file), but the ratio of the results depends on the phantom and diameter of the structure. Therefore, it is not possible to establish correction factors, which would enable the correction of the differences between results obtained for different CDMAM 3.4 phantoms, or to correct the differences between the software applications. Great care must be taken when results of the tests performed with different CDMAM 3.4 phantoms and with different software application are interpreted.

The work was performed in Warsaw, Poland.

\section{References}

1. European Communities. (2006). European guidelines for quality assurance in breast cancer screening and diagnosis. 4th ed. Luxembourg: Office for Official Publications of the European Communities.

2. European Communities. (2013). European guidelines for quality assurance in breast cancer screening and diagnosis. Fourth edition Supplements. Luxembourg:
Office for Official Publications of the European Communities.

3. Young, K. C., Cook, J. J. H., \& Oduko, J. M. (2006). Comparison of software and human observers in reading images of the CDMAM test object to assess digital mammography systems. In M. J. Flynn, \& J. Hsieh (Eds.), Medical Imaging 2006: Physics of medical imaging. Proceedings of SPIE (vol. 6142), 614206-7.614206. DOI: 10.1117/12.653296.

4. de las Heras, H., Schöfer, F., \& Tiller, B. (2013). A phantom using titanium and Landolt rings for image quality evaluation in mammography. Phys. Med. Biol., 58(8), L17-L30. DOI: 10.1088/0031-9155/58/8/L17.

5. BMU. (2012). Development of procedures for nondestructive quality control of phantoms that are used in quality assurance tests according to $\$ 16$, paragraphs 2 and 3 of the German $x$-ray ordinance at $x$-ray systems used for examination of humans UFO-Plan Vorhaben 3608S20001. German Ministry for Environment, Nature Protection and Reactor Security. Braunschweig: PTB. (in German). Available from http://doris.bfs.de/jspui/handle/ urn:nbn:de:0221-2012111310226/.

6. van der Burght, R., Thijssen, M., \& Bijkerk, R. (2010) Manual contrast - detail phantom CDMAM type 3.4. The Netherlands: Artinis Medical Systems BV.

7. Young, K. C., Alsager, A., \& Oduko, J. M. (2008). Evaluation of software for reading images of the CDMAM test object to assess digital mammography systems. In K. C. Young, A. Alsager, J. M. Oduko, H. Bosmans, B. Verbrugge, T. Geertse, \& R. van Engen (Eds.), Medical Imaging 2008: Physics of medical imaging. Proceedings of SPIE (vol. 69131C). DOI: 10.1117/12.770571.

8. Van Metter, R., Heath, M., \& Fletcher-Heath, L. (2006). Applying the European protocol for the quality control of the physical and technical aspects of mammography screening threshold contrast visibility assessment to digital systems. In M. J. Flynn, \& J. Hsieh (Eds.), Medical Imaging 2006: Physics of medical imaging. Proceedings of SPIE (vol. 6142), 614205. DOI: 10.1117/12.650141.

9. Karssemeijer, N., \& Thijssen, M. A. O. (1996). Determination of contrast-detail curves of mammography systems by automated image analysis. In K. Doi, M. L. Giger, \& R. M. Nishikawa (Eds.), Digital mammography (pp. 155-160). Amsterdam: Elsevier.

10. Visser, R., \& Karssemeijer, N. (2011). CDCOM Manual: software for automated readout of CDMAM 3.4 images. Nijmegen, The Netherlands: Radboud University.

11. Klein, S. A. (2001). Measuring, estimating, and understanding the psychometric function: A commentary. Percept. Psychophys., 63(8), 1421-1455. DOI: 10.3758/BF03194552.

12. Young, K. C., Brookes, E., Hudson, W., \& Halling-Brown, M. D. (2012). CDMAM Analyser: Software and instruction manual for automated determination of threshold contrast. Version 1.5.5. Guildford: National Co-ordinating Centre for the Physics of Mammography.

13. Kacker, N. R., Kessel, R., \& Sommer, K. (2010). Assessing differences between results determined according to the guide to the expression of uncertainty in measurement. J. Res. Natl. Inst. Stand. Technol., 115(6), 453-459. DOI: 10.6028/jres.115.031. 